\title{
GuideBeacon Beacon-Based Indoor Wayfinding for the Blind, Visually Impaired, and Disoriented
}

\author{
Seyed Ali Cheraghi*1 \\ Faculty: Vinod Namboodiri, ${ }^{1}$ Laura Walker ${ }^{2}$ \\ ${ }^{1}$ Department of Electrical Engineering and Computer Science, Wichita State University \\ ${ }^{2}$ Envision Research Institute
}

There are currently few options for navigational aids for the blind and visually impaired (BVI) in large indoor spaces. Such indoor spaces can be difficult to navigate even for the general sighted population if they are disoriented due to unfamiliarity or other reasons. This research study presents an indoor wayfinding system called GuideBeacon for the blind, visually impaired, and disoriented (BVID) that assists people in navigating between any two points within indoor environments. It describes the technical challenges faced in designing such a system, the design decisions made in building the current version of the GuideBeacon system, the solutions developed to meet the technical challenges, and results from the evaluation of the system. Results presented in this research study obtained from field testing GuideBeacon with BVI and sighted participants suggests that it can be used by the BVID for navigation in large indoor spaces independently and effectively. 